\title{
CONOSCENZA STORICA E COSCIENZA MORALE IN PIETRO PIOVANI
}

\author{
Fabrizio Lomonaco*
}

\begin{abstract}
RIASSUNTO
Questo saggio discute i volume di libro di Pietro Piovani Conoscenza storica e coscienza morale. Non è una semplice raccolta di saggi (apparsi per la prima volta dal 1959 al 1965), perché c'è un'unità profonda delle pagine data da un interno filo conduttore identificato con la meditazione sul problema della specificità del conoscere, sulle sue conseguenze, tra cui emerge, dunque, la lenta maturazione novecentesca di un senso nuovo della storicità. Nella crisi dei valori assiologici il filosofo napoletano dedica particolare attenzione all'oggetto dello scandalo, l'individualità e pone la questione di partenza comune all'esistenzialismo e alla fenomenologia: la datità primaria dell'ordine, come l'individualità di ordinazione, osservato da Ernst Troeltsch in Der Historismus und seine Probleme. Alle radici del nuovo storicismo in senso critico è il tentativo di dinamicizzare la datità del soggetto nella costitutiva "situazionalità" storica. Nell'individualità come "insolubile mistero" della vita è racchiuso un nucleo di umanità in espansione che il soggetto moderno (leibniziano e non cartesiano) ha lasciato in eredità allo Storicismo (da Schleiermacher a Meinecke) in coerenza con un processo di oggettivazione attraverso il quale l'esistente si autocostruisce quale doveroso-necessario ampliarsi e perfezionarsi.
\end{abstract}

Parole chiave: Conocenza storica. Coscienza morale. Filosofia. Individualità.

\section{ABSTRACT}

This paper discusses about an important Book of Pietro Piovani Historical Knowledge and moral Consciousness. It isn't a simply collection of essays (appeared for the first time from 1959 to 1965), because there is a profound unity in its pages given by the meditation on a specific problem of the knowledge and its consequences, among whom, hence, the slow

* Professore ordinario di Storia della Filosofia Moderna nell'Università degli Studi di Napoli “Federico II”. E-mail: flomonac@unina.it 
maturation in the twentieth century of a new sense of the historicity. In the crisis of axiological values the Neapolitan philosopher pays particular attention to the subject of scandal, the individuality and to the question of the common basis of existentialism and phenomenology: the primary givenness of the order as the individuality ordering, observed by Ernst Troeltsch in Der Historismus und seine Probleme. At the root of the historicism in a critical sense is the attempt to dynamize the givenness of the subject in the constitutive "situational" and historical activity. Individuality as "insoluble mystery" of life is contained a core of humanity expanding the modern subject (Leibnizian and Cartesian) bequeathed to the critical Historicism (from Schleiermacher to Meinecke), in line with a process of objectification through which the existing building up of itself as a duty-must expand and improve.

Keywords: Historical knowledge. Moral consciousness. Philosophy. Individuality.

Il libro di Pietro Piovani Conoscenza storica e coscienza morale non è una semplice raccolta di saggi (apparsi per la prima volta dal 1959 al 1965), riuniti soltanto per uno scopo editoriale o accademico. L'unità profonda delle pagine (inedite quelle raccolte negli ultimi due capitoli) è data da un interno filo conduttore che ha ragione di sussistere per quel «loro naturale ordinarsi intorno a un centro teoretico», identificato con la «meditazione sul problema della specificità del conoscere storico, sulle sue conseguenze, tra cui emerge, da cui deriva, la lenta maturazione novecentesca di un senso nuovo della storicità» ${ }^{1}$. In brevi ma efficaci note autobiografiche del 1972 l'Autore riconoscerà che «l'interesse al mondo storico» poteva considerarsi «istintivamente, proprio della mia forma mentis», favorito «dall'accoppiamento idealistico di filosofia e storia, a cui negli anni giovanili sono stato educato» ${ }^{2}$.

A metà degli anni Sessanta il suo impegno teorico e storiografico consolidava gli esiti delle analisi critiche sul giusnaturalismo, maturate nella monografia del 1961 (Giusnaturalismo ed etica moderna), una magistrale

\footnotetext{
1 Così Piovani nella Prefazione a Conoscenza storica e coscienza morale, Napoli, Morano, 1966, p. 7 (d'ora in poi con la sigla CSCM).

2 [Intervento nell'inchiesta:] Parlano $i$ filosofi italiani, a cura di V. Verra, in «Terzoprogramma», n. 3 (1972), p. 160.
} 
opera di "filosofia storica" sul diritto naturale dalle origini al moderno che illustrava con finezza le ragioni del passaggio dall'antica cosmologia alla moderna antropologia, fondata sull'etica individuale-interindividuale; un vero e proprio luogo di svolta della riflessione piovaniana che, in coerente evoluzione teorica dei temi selezionati, inaugurava (anche dal punto di vista cronologico) una stagione nuova del pensiero, teso ad abbandonare l'ultima rinnovata forma di naturalismo (il "diritto di natura"). Dopo gli studi giovanili, impegnati nello studio di questioni filosofico-giuridiche attinenti alla norma, ripensata alla luce di profili propri di un ontologismo critico al punto da ridefinire quelle questioni nel senso e nella prospettiva della "normazione" e in funzione della societas ${ }^{3}$, nasceva un interesse per la storia e la società degli individui. Il tutto orientava a un «ripensamento dello storicismo» ${ }^{4}$ alla luce di quelle filosofie del Novecento europeo che avevano contribuito a identificare nelle idee di individualità e di esistenza i centri tematici gravitazionali del pensiero contemporaneo. Nella crisi dei valori assiologici il filosofo napoletano dedica particolare attenzione «all'oggetto dello scandalo, l'individualità» ${ }^{5}$ e pone la questione di partenza comune all'esistenzialismo e alla fenomenologia, richiamando la «datità primaria non dell'ordine, ma dell'includente ordinante ${ }^{6}$ a commento di uno dei padri dello storicismo tedesco, Ernst Troeltsch, e delle sue pagine su Der Historismus und seine Probleme, la cui diffusione in italiano deve molto al magistero piovaniano. Alle radici del nuovo storicismo in senso critico, antidealistico, antihegeliano e anticrociano è il tentativo

\footnotetext{
3 Mi riferisco alle pagine di Normatività e società (1949), ora in P. Piovani, Per una filosofia della morale, a cura di F. Tessitore, Milano, Bompiani, 2010 (d'ora in poi si cita con la sigla PFM), trad. tedesca, hrsg. von M. W. Hebeisen mit einer Einführung von G. Cacciatore und F. Tessitore, Schweizerischer Wissenshafts-und Universitätsverlag SWUV, 2010 II. Abteilung, Band 6. Per un aggiornamento delle letteratura critica rinvio alla Bibliografia degli scritti su Pietro Piovani (1948-2000), a cura di P. Amodio, Napoli, Liguori, 2000, alla Bibliografia degli scritti di storia della filosofia di Pietro Piovani, a cura di G. Giannini, in P. Piovani, Indagini di storia della filosofia. Incontri e confronti, a cura di G. Giannini, con una nota di F. Tessitore, Napoli, Liguori, 2006, p. XLIX-LXI, nonché alla Bibliografia degli scritti su Pietro Piovani (2000-2007), a cura di P. Amodio, in «Archivio di storia della cultura», XXI (2008), p. 361-371.

${ }^{4}$ Così F. Tessitore, L'«itinerario» di Pietro Piovani, in Id., Contributi alla storia e alla teoria dello storicismo, vol. V, Roma, Edizioni di Storia e Letteratura, 2000, p. 425.

${ }^{5}$ Cfr. Id., L'etica assenzialistica di Pietro Piovani, in «Filosofia», XXXI1 (1981), 4, p. 487

${ }^{6}$ CSCM, p. 163.
} 
di dinamicizzare la datità del soggetto nella costitutiva "situazionalità" storica. Nell'individualità come «insolubile mistero» della vita è racchiuso un nucleo di umanità in espansione che il soggetto moderno (leibniziano $\mathrm{e}$ non cartesiano) ha lasciato in eredità allo Historismus (da Schleiermacher a Meinecke) in coerenza con un processo di oggettivazione attraverso il quale l'esistente si autocostruisce quale «doveroso-necessario ampliarsi e perfezionarsi» ${ }^{7}$. In questi due verbi, in queste due "azioni" si intersecano l'ideale idealistico e quello illuministico in uno sfondo teorico proprio di un pensiero (piovaniano) che, contro le rinnovate pretese ontologiche dei diversi e ambiziosi esistenzialismi (quelli a vocazione neometafisica più $o$ meno camuffata), si pone invece come filosofia degli esistenti.

Alla demitizzazione di ogni forma astratta di universalismo corrisponde l'esperienza della coscienza individuale che, nel suo costitutivo esistere storico, esprime l'interrogarsi sull'autocomprensione e sull'«oggettivizzabilità inconscia» comune alle riflessioni dello psichiatra Jung (Il divenire della personalità) e del l'esistenzialista Jaspers (Introduzione alla filosofia) dei quali Piovani sottolinea gli itinerari ideali, tutti positivamente sottratti al pericolo di cadute nel naturalismo di una psicologia scientistica. Il che consente di notare che gli originali richiami storicistici alla nozione di anima quale «centro profondo dell' individualità» (Meinecke) e «originaria unità di coscienza e di conoscenza», attitudine «potenziale degli umani a mediare pensieri e azioni nell'ossequio autonomo a una energia universalizzante», confermano l'esigenza di studiare le manifestazioni del conoscere e dell'agire di ogni individualità nel suo «profondo insondabile» da riferire al pluralistico ordine delle anime e non più alla tradizionale "anima del mondo" 8 . Se tale riflessione

\footnotetext{
7 Ivi, p. 164

8 Ivi, p. 167, 168, 169. Nel pensiero antico da Aristotele ad Agostino e in quello medievale (con s. Tommaso) si rinnovano le esigenze di difesa dell'autonomia personale dell'io, perché «la collocazione dell'anima dell'uomo nell'anima del mondo sembra (...) esigere l'esclusione d'un mondo di anime da sacrificare al monismo di un universo fisico-etico concettualmente sistemato» (ivi, p. 213). All'«ultimo Jaspers» guarda la sensibilità critica del Piovani che intende anche distaccarlo dallo «sfondo del generale e generico 'esistenzialismo'»: si veda la Nota pubblicata nel «Giornale critico della filosofia italiana» del 1965, poi in P. Piovani, Scandagli critici, a cura di G. Di Costanzo e F. Lomonaco, Napoli, Morano, 1986, p. 129-131.
} 
documenta, nel tratto breve di un'acutissima diagnosi, l'interesse per i risvolti psicologici di una filosofia della morale, preoccupata di teorizzare i propri princìi teorici ${ }^{9}$, l'impegno prevalente in queste pagine del 1966 (credo non a caso ristampate nel 1972) è teso a definire i contrassegni fondamentali dell'individualità a partire dalla riconosciuta dimensione dell'alterità. Del resto la conclusione del solipsismo (per parafrasare un acuto saggio piovaniano del 1949) indica già un approdo maturo al rifiuto del soggetto empirico. L'oggettivazione dell'io contiene già un invito alla coesistenza, facendo trasparire una fondamentale verità: l'esistere è coesistere, non c'è esistenza che non sia coesistenza. In essa l'obiettivazione dell'io «riesce ad avvicinare l'io all'altro, mostrando che l'alterità non è pura cosalizzazione ${ }^{10}$. L'esistenza in quanto coesistenza non può essere negazione dell'individualità che si realizza nella tensione ultraparticolaristica. Gli esistenti non sono mai considerati come qualcosa di definitivamente compiuto, perché l'individualità è un individuarsi, un' «incompiutezza da compiere ad infinitum ${ }^{11}$. Caduto l'io come soggettività pura, il soggetto deve abbandonare il proprio desiderio di essere sempre e tutto volente e titolare di un'illimitata libertà di agire

\footnotetext{
${ }^{9} \mathrm{Mi}$ riferisco naturalmente alle pagine dei Principi di una filosofia della morale (1972), in PFM; trad. tedesca, hrsg. von M. W. Hebeisen, mit einer Biographie von F. Tessitore, und mit einer bibliographie von F. Tessitore und G. Acocella, Schweizerischer Wissenshaftsund Universitätsverlag SWUV, 2010 I. Abteilung, Band 1.

${ }^{10}$ CSCM, p. 164. In una Nota di autopresentazione dell'opera (il cui originale dattiloscritto si conserva (ora nella Biblioteca della Fondazione "P. Piovani per gli studi vichiani" di Napoli) tra le carte autografe e il carteggio di riferimento a CSCM si legge «Il conoscere storico è qualcosa di più della metodologia propria della scienza storica, sebbene da tale metodologia concreta debba sempre prendere umilmente lezione, è la forma del conoscere individualizzante (...), una filosofia di individualità al plurale), intese come realtà realizzantisi in una espansione implicante il dovere del loro reciproco rispettoso coesistere (...) nelle individue tensioni, in cui è il superamento dell'immediatezza come empiricità (empiricità che si nega all'esperienza) e come velleità di clausura solipsistica)» (Nota di autopresentazione di CSCM, in "Atti dell'Accademia di Scienze morali e politiche della Società Nazionale di Scienze, Lettere ed Arti in Napoli», LXXVII, 1966, p. 549).

${ }^{11}$ CSCM, p. 166. «Proprio l'adesione a quell'esistenzialismo di modulazione jaspersiana ha scritto Perrucci - fa di Piovani un attento scrutatore della storia che si individualizza nel sua rivolgersi ad ogni azione attestante l'umanit'a dell'uomo, che riconosce tra le formedi civilit'a l'irripetibile forma di cui ogni uomo d'a prova della sua propensione all'infinito» (A. Perrucci L'etica delle responsabilità. Saggio su Pietro Piovani, presentazione di F. Tessitore, Napoli, Liguori, 2007, p. 32).
} 
come vuole. La sua struttura è de-ontologica in quanto deficitaria. Nella razionalità l'uomo giunge a piena consapevolezza della sua origine irrazionale e, perciò, rinuncia a trovare un fondamento, consapevole che il proprio fondamento non è perché è mancanza. Gli uomini avvertono la loro imperfezione ma con l'ansia gravosa del perfezionamento, a cui cercano di rispondere l'individuazione e la individualizzazione dell'altro, fondando agostinianamente la società non solo «in interiore homine ma inter homines», in quanto agonistico impegno a misurare lo sforzo che costa il lavoro di ogni soggetto, scopertosi nella sconcertante condizione di «volente non volutosi». Non dimentico dell'elaborazione maturata nelle Linee di una filosofia del diritto (1958) $)^{12}$, Piovani riprende tale motivo nella consapevole ricerca di un'aperta dialettica tra la datità del soggetto e la fatticità oggettiva. Nella filosofia moderna svanisce la tradizionale antitesi di soggetto e di oggetto, perché l'individualità si realizza nel confronto con l'oggettività rinvenuta all'origine della sua esperienza di sé. L'oggettivo diviene un quid compartecipe della medesima soggettività che, per rimanere se stessa, deve obbiettivarsi in una realtà di vita che la confermi e rinsaldi. Qui è possibile cogliere la funzione che la fenomenologia ha svolto nella cultura filosofica moderna, il valore del suo disegno alternativo al soggettivismo antico e moderno, «l'esemplare testimonianza di un pensiero inesauribilmente disposto a mettersi continuamente in discussione in ogni sua pagina, in una apertura e riapertura di problemi che non è funambolismo dialettico, ma è tensione verso una volontà di comprensione rinnovata; non è offerta di formule di facile consumo, ma proposta di uno 'stile' di investigazione, di un metodo di osservazione». Questa osservazione si raccoglie nel saggio su Ricognizione dell'individuale, scienza storica, filosofia italiana, interesse fenomenologico, per sostenere che il linguaggio fenomenologico, al di là di falsi rigorismi, delle rigide schematizzazioni e di un'astratta logicizzazione scientista, partecipa degli sviluppi della filosofia italiana (e dentro di essa a quella piovaniana che si appresta ad essere una filosofia della morale) con la ricerca dinamica dell'autentico che vive nella

\footnotetext{
${ }^{12}$ P. Piovani, Linee di una filosofia del diritto (1958), poi in PFM, p. 505-506 (d'ora in poi si cita con la sigla LFD; trad. tedesca, hrsg. von M. W. Hebeisen mit einer Auswahlibliographie der Sekundärliteratur von P. Amodio und M. W. Hebeisen, Schweizerischer Wissenshaftsund Universitätsverlag SWUV, 2010 II. Abteilung, Band 7).
} 
vita di «ogni individuato fenomeno intenzionalmente seguito e compreso» con «un senso esasperato della mobilità di ogni vivere» ${ }^{13}$. Il linguaggio fenomenologico può favorire, nelle sue interpretazioni italiane (Il Diario di Paci, studioso di Vico e di Croce), la nascita di un clima teorico disposto ad abbandonare ogni logicizzazione. Così osserva Piovani, avvertendo l'«astoricità» di Husserl, coerente al «suo programma, almeno iniziale»e citando della Crisi le pagine del capitolo su «La via di accesso alla filosofia trascendentale fenomenologica attraverso la riconsiderazione del mondo della vita già dato». Pretendere di conoscere il nostro costitutivo essere è porsi l'acuto interrogativo: "Che siamo noi in quanto soggetti che compiono l'operazione di senso e di validità della costituzione universale, noi che, nella comunità dei soggetti, costituiamo il mondo come polisistema, cioè come formazione intenzionale della vita in comune?» ${ }^{14}$. Già Capograssi in l'Analisi dell'esperienza comune rifletteva sulle laceranti difficoltà della civiltà contemporanea per la complicata separazione della speculazione filosofica dalla vita e ne portava alla luce le nefaste conseguenze per una comprensione effettiva della vita vissuta dall'individuo ${ }^{15}$. Una separazione spiegata dal fatto che la vita che si pone come oggetto della speculazione filosofica non è quella dell'individuo concreto, costretto a sopprimere se stesso proprio per poter vivere, ma quella di un'individualità che si trascende. Per Piovani luogo di riflessione non è più la trascendenza o una provvidenza indifferente al vissuto umano. La crisi del soggetto singolo quale pura essenza di pensiero irrelato esprime l'esigenza di una logica non astratta né assoluta, legata alla concretissima praticità della vita, a sua volta non assolutizzata che prende l'avvio dalla realtà dell'io come individuo agente. Maturate nella critica dell'idealismo, queste tesi si saldano ai primi interessi piovaniani per la filosofia dell'azione (Blondel) nella direzione di una filosofia della vita, lontano dalle vuote formule del «vitalismo attivistico», perché interessata a coniugare il pensiero con la vita, a immettere il primo nel concreto divenire della seconda.

13 CSCM, p. 72-73.

${ }^{14}$ Ivi, p. 59, 66, 73, 71-72.

${ }_{15}$ G. Capograssi, Analisi dell'esperienza comune (1930), poi in Id., Opere, Milano, Giuffrè, 1959 , p. 5-8. 
Per tale impegno al concetto tradizionale di persona (consapevole con Vico e Nietzsche della vita tragica espressa nell'antico significato di maschera) si sostituisce quello dinamico di personalità. Questa che diviene e «si fa» non è il prodotto di un rapporto discorsivo tra due momenti interni al sé dell'individuo; è confronto e scontro insieme nei riguardi di un'originaria datità che il soggetto riscopre dapprima in sé come limite alla volontà e assume poi come punto di partenza della propria tensionalità. Il fatto della coesistenzialità dell'esistenza, trasfigurato in dovere diventa il punto di partenza per uno sforzo teso all'oggettivazione sul piano del sociale delle proprie e altrui volizioni. L'individuo rinviene il segno della propria finitezza, della propria incompletezza e però, insieme, avverte la vocazione alla «completezza» che gli impedisce di chiudersi in sé. Della moderna personalità dinamica Piovani identificava l'autenticità in un concetto di auto-determinazione, sviluppatosi, nello storicismo critico (da Dilthey a Troeltsch, da Droysen a Meinecke), sul piano dell'interesse biografico-storico. L'individualità è la voce di un essere che si scopre reale nell'esistenza storica, impegnato nella progressiva realizzazione di sé e di ciò che non è ancora, al punto che la «realità dell'essere (...) la sua positività essenziale» sono la «sua negatività esistenziale» ${ }^{16}$. Lo storicismo aiuta a vedere come ogni ricerca sull'umano e sulle sue produzioni debba tenere conto del portato dell'azione dell'individualità che, personalizzandosi, plasma, insieme a se stessa, anche gli altri oggetti dell'esperienza in un'attività «spirituale» che trasforma ciò che incontra. Accettarsi come coesistente significa riferirsi a una concezione filosofica per la quale le esigenze e i doveri dell'individuo tendono a chiarire i limiti e le ragioni dell'essere storico dell'uomo. All'individualità, intesa diltheyanamente come esperienza vissuta, occorre riconoscere una tendenziale capacità di farsi portatrice di valori incontenibili nel finito. Il volere umano si infutura nelle infinite forme dell'Erlebnis, in ogni esperienza pienamente vissuta dall'uomo che supera l'accidentalità nella storia. La considerazione jaspersiana «del vario e del molteplice» (nella citata Psicologia delle visioni del mondo) contribuisce a realizzare un'autentica umanità, la «comprensione del poliformismo individuato nella pluralità delle esistenze,

${ }^{16}$ CSCM, p. 179. 
liberando dalla tendenziale prepotenza dell'unità contenutistica, che induce all'uniforme e all'uniformismo» ${ }^{17}$. L'esistenzialismo che si afferma nel Novecento vuole rispondere all'esigenza di un recupero filosofico delle esistenze individuate, prospettando un nuovo interesse per la storicità: il senso del reale non può essere colto fuori dalle trame complesse del concreto contesto storico in cui si articola la sua stessa vita. Di fronte agli esistenti che reclamano d'esser compresi non in nome di un'astratta ragione ma nelle loro individuate ragioni e nella resistenza alla riduzione integrale all'essere unificante, la filosofia si individualizza, dilatando la forma di conoscenza e la logica dei suoi radicali ripensamenti. La tendenza all'«esistenzializzazione» è propria del pensiero contemporaneo, come risulta ora «indirettamente ora direttamente dalle filosofie dell'esistenza, dell'azione, dell'esperienza, intese quali autentiche rappresentanti dello spirito intimo del filosofare moderno e della sua sostanziale vocazione» a farsi «faticosa maieutica dell'individuale» ${ }^{18}$. L'esistenzialismo è la negazione di ogni ordine storico dato, la fine di ogni filosofia della storia, di ogni teleologia come provvidenzialistico ordine includente e, insieme, la caduta dell'aggiornata cosmicità fisico-metafisica. L'esistenza può essere colta solo nella storia, la storia cessa di essere storia dell'universo, per diventare la storia di ogni esistente in divenire. L'incontro di Piovani è tra l'«esistenzializzazione» dello storicismo e la «storicizzazione» dell'esistenzialismo, ossia la dissoluzione del dilemma «fra una storia senza esistenza e un'esistenza senza storia», favorita da Kierkegaard contro la Weltgeschichte di Hegel19. Disperso nell'illimitato e senza "dimora", l'uomo è stato costretto a ripiegare sull' angoscia della propria imperfezione e situazione esistenziale, secondo quanto si apprende dalle note diagnosi presenti nello Jaspers della Psicologia delle visioni del mondo e nello Heidegger dell' «analitica esistenziale» (in Essere e Tempo), indicati dal Piovani come i massimi esponenti dell'esistenzialismo, perché avvertiti che non è possibile guardare e scandagliare l'esistente senza «guardare allo storico e viceversa $»^{20}$.

\footnotetext{
${ }_{17}$ Ivi, p. 176, 235; ma si vedano anche le p. 149-152 sul «poliformismo della storia».

${ }_{18}$ Così nella Nota di autopresentazione di CSCM, cit., p. 550 e CSCM, p. 65.

${ }^{19}$ CSCM, p. 188, 189.

${ }^{20}$ Ivi, p. 108
} 
Piovani può, così, concordare con Meinecke, coniugando individualità e svolgimento, per originale interesse a «interne forze formatrici», secondo un lessico che richiama Leibniz e Humboldt, teorici della moderna connessione tra l'individuale e l'universale, compito dello storico, condiviso da un loro notevole interprete novecentesco, Ernst Cassirer, trattando della filosofia delle formesimboliche ${ }^{21}$. Su tale dimensione il pensiero del Novecento ha insistito per delineare la condizione umana e le strutture esistenziali che la costituiscono in una visione dinamica, attenta al sistema-sistemazione delle azioni individuali e coerente con la definizione della «lingua come istituto» degli studiosi fiorentini ammirati e frequentati dal Piovani, specialmente Giovanni Nencioni e Giacomo Devoto, «sottile moralista», teorico di quella «civiltà di parole» che è «ricerca d'umanità», come il filosofo morale acutamente suggeriva in un fine intervento del 1965 dopo le acute pagine scritte su Mobilità, sistematicità, istituzionalità della lingua e del diritto ${ }^{22}$. Nel 1966 il tema ritorna quale possibile rappresentazione del radicale mutamento degli indirizzi spirituali che, nella metà del Novecento, hanno favorito l'affermazione di motivi antipositivistici e storicizzanti rispetto al modello matematizzante. Agli eccessi di quest'ultimo corrispondono i limiti di una cattiva generalizzazione che rischia di eludere la «conoscenza precisa di quell'individuale senza di cui la sua funzione individualizzante viene meno» ${ }^{23}$. Ma la tendenza ad esprimere immediatamente il vissuto non è esente dai rischi del naufragio nell'ineffabile e nell' «angoscia» dell'inesprimibile. Da questo punto di vista il confronto critico tra $\mathrm{i}$ «linguaggi» della scienza può raggiungere una significativa alleanza, facendo valere istanze tipiche del linguaggio

\footnotetext{
${ }^{21}$ Ivi, p. 60 e note.; sull' «individualismo universalistico di Humboldt si veda anche CSCM, p. 116.

22 P. Piovani, Civiltà di parole (1965), poi in Id., Margini critici, presentazione di F. Tessitore, Napoli, Bibliopolis, 1981, p. 67; Id., Mobilità, sistematicità, istituzionalità della lingua e del diritto (1962), poi in Id., La filosofia del diritto come scienza rigorosa, Milano, Giuffrè, 1963, p. 103-193. Si veda la recensione del Nencioni in "Lingua nostra», XXIII (1962) 4, p. 97-102 e le Note dedicate, nel «Giornale critico della filosofia italiana» del 1963 e 1965, alla «linguistica storicistica» di Devoto e Terracini, poi in P. Piovani, Scandagli critici, cit., p. 46-47, 120-121, 123-124.

${ }^{23}$ CSCM, p. 16. Questo motivo è stato messo opportunamente in risalto nella recensione a CSCM di D. Grimaldi, in «Il Pensiero», XII (1967) 3, p. 356.
} 
scientifico e, insieme, indicazioni metodologiche, impegnate a evitare comode ma generiche astrazioni. Contro la teoresi del puro pensiero si afferma la filosofia della vita con il suo «linguaggio storicizzante in quanto individualizzante o volenteroso di individualizzare» a vantaggio della «logica del pensiero concretantesi nell'agire» e con sviluppi in senso antipositivistico perfino di suggerimenti del naturalismo positivistico come accade nel Bergson letto e studiato dal Piovani sin dagli anni giovanili, riproposto, nel testo del 1966, accanto alla «filosofia» nuova di Cassirer, due esempi notevoli di «un'antropologia filosofica rinnovata», fatta non più di concetti ma di concezioni ${ }^{24}$. Essa si nutre non più di oggetti ma di forme necessarie all'azione che, contro il formalismo di forme astratte, costituisce il moderno processo di oggettivazione e di esperienza vissuta. In proposito l'autore richiamato e citato è il Dilthey della Critica della ragione storica, impegnato ad accordare significativa fiducia alla conoscenza storica, per poter conoscere quell'esperienza rivissuta nel divenire tensionale ad infinitum. La vocazione antropologica dell'epoca contemporanea, richiamata da note e citate pagine di Scheler, esprime una volontà di concretezza contro le generalizzazioni coincidente con l'interesse nuovo per la storia al punto tale che la nota formula di Antoni va ripensata e ribaltata in dalla sociologia allo storicismo ${ }^{25}$. La storia è, allora, una nuova filosofia in quanto antropologia, disposta a comprendere l'interiorità delle coscienze

${ }_{24}$ CSCM, p. 14, 13, 145, 146, 143, 144. Il tema è al centro anche di acute riflessioni sulle «scienze umane» e, in particolare, su «Gli studi di etnologia, antropologia, sociologia, psicologia, le indagini sulla preistoria» a proposito del noto studio del Cantoni sui primitivi: si veda la Nota pubblicata nel «Giornale critico della filosofia italiana» del 1964, poi in P. Piovani, Scandagli critici, cit., p. 59-60.

25 Ivi, p. 128-129, 134-135, 136. A «Dilthey e la comprensione del mondo umano» è dedicata una Nota pubblicata nel «Giornale critico della filosofia italiana» del 1966, poi in P. Piovani, Scandagli critici, cit., p. 157-158. "La filosofia più consapevolmente e sinceramente moderna è tutta tendenzialmente "antropologica" perché non crede più a una conoscenza totale, contemplativa di realtà da identificare in una loro essenza fisica o logica data (...) ma vede il complesso delle esperienze in formazione come rappresentazioni, con le quali il pensiero deve di volta in volta misurarsi non attraverso concetti bensì mediante concezioni» (ivi, p. 144). Il tema è centrale nell'analisi di A. M. Nieddu, Normatività Soggettività Storicità. Saggio sulla filosofia della morale di Pietro Piovani, Napoli, Loffredo, 2001, p. 105-107. Per il rapporto Piovani-Dilthey, cfr. i saggi di G. Cacciatore, in part. Storicità e Historimus, in L'opera di Pietro Piovani, a cura di F. Tessitore, Napoli, Morano, 1991, p. 347-398. 
indagate» al riparo dalle «seduzioni dello psicologismo scientistico, abile a classificare e sezionare, incapace di ricostruire e penetrare $\nu^{26}$. Intesa la lezione della «conoscenza storica», il filosofo moderno, erede di Vico, deve identificare la natura dell'uomo nella sua storia e, perciò, fondare «un verace storicismo, integralmente anaturalistico e ateologistico». In tale prospettiva la demitizzazione bultmanniana è vista da Piovani come un bisogno di liberarsi, assai più che dalle «presunte scorie mitiche» di cui si è caricato il sacro, dall'involucro più antico e vasto, di una concezione del «cosmo come universale luogo naturale-sovrannaturale contenente l'uomo». Perciò può essere accolta la lezione di Löwith, esperta di esistenzialismo, fenomenologia e storicismo che radicalizza la distinzione tra natura e contingenza umana nel mondo con «scacco esistenziale»e in netta distanza dalle due convinzioni, antica (cosmologica) e premoderna (creazionistica e teologica $)^{27}$. Le esperienze della religiosità più intima, che muovono dalla Riforma e da Pascal e rivivono in Blondel e Bultmann, si alleano a definire il nesso tra individualità e storia, dove la prima è garanzia di presenza umana e religiosa in un itinerario che Piovani segnala con particolare finesse, attento alle esperienze letterarie (Mann) e filosofiche (il Simmel de Il conflitto della civiltà moderna). Il nuovo ordine è l'interiorità della coscienza che l'umanesimo fonda, abbandonando il personalismo tomistico e avvalendosi della «mediazione agostiniana del socratismo cristiano», lungo un percorso che vede la distinzione petrarchesca dell' individuo dal mondo e il richiamo all'interiorità del «rude agostinismo di Lutero» e del fine agostinismo di Pascal ${ }^{28}$. L'uomo conosce veramente se stesso, solo se libera la propria storia dal fisicismo metafisico, seguendo, anche in questo, l'insegnamento di Dilthey, secondo il quale tale autoconsapevolezza è «un modo della coscienza di sé»» ${ }^{29}$. Ad essere messo in risalto è l' «intimità» dell'uomo dai tempi del Petrarca a

${ }_{26}$ CSCM, p. 146, 147, 199. Sul momento individuale non classificabile del conoscere storico cfr. ivi, p. 223.

27 Ivi, p. 187, 193, 186, 187.

28 Sul tema si veda il magistrale studio di G. Lissa, Anti-ontologismo e fondazione etica in Pietro Piovani, Napoli, Giannini, 2001, p. 191. In proposito R. Crippa ha notato il convergere della «blondeliana tradizione e lo humiano custom» (Recensione a CSCM, in «Giornale di Metafisica», XXIII, 1968, 2-3, p. 334).

${ }^{29}$ CSCM, p. 197. 
quelli di Montaigne ${ }^{30}$, il «destarsi dell'interiorità», sottolineato anche da Meinecke, polemico contro Croce a proposito dell'individualità, posta quale condizione del significato storico in antitesi con la restaurazione matematico-universalistica, perché la vera scienza storica è estranea alle classificazioni ${ }^{31}$. L'uomo, infatti, si distingue non all'interno di uno schema tipologico generale, ma per essere un unico nella sua specificità che accade una volta sola, non un'unità ripetuta all'infinito.

Piovani considera evento fondatore della modernità la scomparsa dell'immagine tradizionale (medievale) del cosmo che, costruito sull'antica alleanza di fisica e metafisica, aveva prospettato un ordine di perfette armonie, all'interno del quale si collocava ogni esistente, partecipe della perfezione in quanto parte di un tutto inglobante. Ma quando svanisce tale perfetta totalità, crollano tutte le certezze e si produce il distacco tra l'Essere infinito e l'esistente finito che, gettato nel mondo, diventa cosa tra cose. La coscienza umanistico-rinascimentale nasce dalla nuova e autonoma comprensione dell'individuo umano, rivolta a un nuovo soggetto-oggetto, perché nuovo è l'uomo indagato e indagante. La meccanica celeste che disintegra i cieli perfetti nelle rotanti armonie, ereditate dal Timeo, non ritrova più in mezzo ai frantumi della vecchia cosmologia l'uomo aristotelico. Dissolta la cornice universalistica, solida e rassicurante nella sua plurisecolare continuità, il conoscente, conosciuto nella sua pienezza, è considerato un universo da esplorare, cessa di essere un accidente che la logica deve essenzializzare. Il pensiero da cosmologico si fa umanologico. Il nuovo uomo microcosmico ha una razionalità diversa; è assai più ragionante che razionale. All'orizzonte cosmico si sostituisce quello umano, ricco di ogni forma di vita storica che «è quel che è e non c'è altro modo di conoscerla per quel che è che seguirla nel suo formarsi embrionale e corposo, lungo l'itinerario complesso del suo esistere, che è tutto un transformare» ${ }^{32}$. Il rifiuto di ogni forma di cosmologia deve essere

\footnotetext{
${ }^{30}$ Ivi, p. 92. Intimità di Montaigne è il titolo di un acuto intervento pubblicato nel 1970, poi in P. Piovani, Indagini di storia della filosofia. Incontri e confronti, a cura di G. Giannini, con una nota di F. Tessitore, Napoli, Liguori, 2006, p. 421-424.

31 A «Meinecke nel ripensamento dello storicismo» è dedicata una Nota pubblicata nel «Giornale critico della filosofia italiana» del 1963, poi in P. Piovani, Scandagli critici, cit., p. 31-32.

32 CSCM, p. 81, 206. Sul tema sono da leggere le ricostruzioni di W. Ghia, Il pensiero di Pietro Piovani, Genova, E.C.I.G, 1983, p. 37-67.
} 
deciso e radicale, perché non è possibile, come vorrebbe Alberto Caracciolo (uno dei più stimati interlocutori del Piovani), imboccare entrambe le strade: conservare la «cosmologia tradizionale»e, al tempo stesso, accogliere un nuovo concetto di «interiorità cosmica» in cui si sviluppano «le individue esistenze coesistenti secondo il ritmo di una loro intima legge coscienziale». E' dopo Hegel e la sua concezione metafisico-religiosa che l'esperienza religiosa conosce la sua autonomia e, insieme, la rivela al reale storico secondo le lezioni di Simmel e Scherer, Dilthey e Troeltsch fino al Meinecke, letto da Caracciolo che propone interessanti argomenti di "teologia negativa" nel suo Valore religioso e valore morale (1960) che aiutano a capire come nel tessuto connettivo delle situazioni l'essere coscienzializzato entri in relazione alle coscienze, desostanzializzandosi ${ }^{33}$.

Nella coscienza individuale va cercato il nuovo universale. La filosofia nuova deve prepararsi a esplorare i non limitanti limiti dell'individualità, accogliendo quanto c'è di kierkegaardiano nell'esistenzialismo di Jaspers o di Heidegger; deve familiarizzare col «singolo» del filosofo di Copenaghen, studiando gli essenziali aspetti in cui esso non è singolaristico ma fautore dell'universalità insita nell' individuale. E' una direzione di pensiero complessa e articolatissima che può avvicinare posizioni storicistiche anche a motivi essenzialistici ma con formulazioni teoriche radicalmente differenti. Piovani è, infatti, acuto nell'osservare che "se la mancanza di confidenza "metafisica" con l'individuale induce ancora a confondere Kierkegaard con Stirner, la meditazione parallela sulla lezione di un Meinecke e sulla lezione di un Heidegger, pur quando risulti assai viva negli interessi dimostrati e suscitati, non arreca il contributo di novità che potrebbe arrecare» ${ }^{34}$.

L'«originalità» dell'esistente individuale è compresa nel suo essere universale che vive nell'individualità come universalizzazione, incarnato come tensione costitutiva della personalità verso il perfezionamento della vera umanità dell'uomo ${ }^{35}$. Il soggetto universalizzante non è, ma si fa

33 CSCM, p. 121, 122, 107, 109, 111-112, 116.

34 Ivi, p. 124.

${ }^{35}$ Giuseppe Cantillo ha sottolineato la complessità di questo fondamentale tema piovaniano, mettendo in rilievo la complicata rete di condizionamenti che oppone resistenza alle volizioni del soggetto: «Non solo io non mi sono voluto quanto al nudo fatto di esistere. 
universale, guadagna la propria universalità lontano da ogni residua Iità e rappresentazione determinata, riconoscendosi non oggettivo, bensì da oggettivare, senza vedere annullate le sue contraddizioni in artificiose coerenze. Da qui il richiamo a Merleau-Ponty (il fenomenologo francese eterodosso che Piovani privilegia, riconoscendogli l'originale attenzione al comportamento dell'io e alla sua coscienza percettiva) che ha garantito una conoscenza dinamica per sottolineare la volontà di trovare dentro l'uomo ciò che è umano e più che umano (Il metafisico nell'uomo), l'universalità intrinsecata nell'individualità ${ }^{36}$.

Per tutto ciò le risposte alla «ricognizione dell'individuale» non sono più quelle delle distinzioni certe e statiche tra universale e particolare. Universalismo e particolarismo sono entrambi parziali: se il primo tralascia di considerare l'individualità del soggetto, il particolarismo non coglie la tensione universale che lo fa essere profondamente se stesso, nel momento in cui ambisce ad andare oltre l'immediatezza: «Per essere se stesso, deve sempre essere più di se stesso: se fosse soddisfatto del proprio essere, non sarebbe nella sua essenza caratteristica ${ }^{37}$. Compito del nuovo filosofare è comprendere l'individuale oltre universalismo e particolarismo che possono rivelarsi poli distinti e, insieme, alleati in infide salvezze individuali. La filosofia moderna aiuta a intendere che il particolarismo umanistico non è semplice tendenza a considerare assolutamente autonoma ogni realtà fornita di valore particolare; è qualcosa di più profondo proprio per il suo voler essere esaltazione di individualità non particolaristiche ${ }^{38}$. Il valore

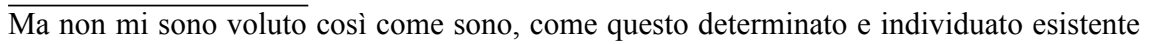
qui e ora, con un determinato patrimonio, genetico, psichico, culturale, inserito in una determinata tradizione, in una determinata storia, in una determinata situazione: con una serie di condizionamenti e di vincoli che non vorrei avere, ma senza i quali, peraltro, non sarei quale sono, e quale sono dato a me stesso, assieme al mondo circostante, in una originaria evidenza intuitiva» (G. Cantillo, Per una «filosofia degli esistenti»: lo storicismo esistenziale, in L'opera di Pietro Piovani, cit., pp. 488-489, poi in Id., Eccedenza del passato. Per uno storicismo esistenziale, Napoli, Morano, 1993, p. 311-374).

36 CSCM, p. 90-91. Su questo aspetto e con riferimento al fenomenologo francese si veda l'acuta recensione di R. Crippa a CSCM, cit., p. 335.

${ }^{37}$ CSCM, p. 152. Sulla «tensione all'universale» sono molto utili le acute pagine di E. Nuzzo dedicate alla storia delle idee tra conoscenza storica e riflessione morale nella silloge su Storia ed eredità della coscienza storica moderna. Tra origini dello storicismo e riflessione sulla conoscenza storica nel secondo Novecento, Napoli, Liguori, 2007, parte II, capp. III e IV (p. 101-164). 
dell'individualità non si identifica con l'«arbitrio, non è sregolatezza di capriccio particolare, perché è documentazione della formazione di persone che sono tali in quanto sono capaci di superare il singolarismo dell'arbitrario». L'individuo del particolarismo umanistico sa di non poter fare della propria singolarità la "regola dell'universo" come nella diversità e analogia delle posizioni insegna il pensiero moderno, riconoscendo con Leibniz le leggi proprie dell'individualità agente e con Vico la storicizzazione dell'universale: «La filosofia moderna aveva bisogno di (...) una microfilosofia che aiutasse la conoscenza di una più approfondita dimensione dell'umano: la storia come conoscenza individuante dell'individuale è la scienza che permette questa ricognizione ${ }^{39}$. Ad affrontare, in termini teorici maturi, il problema dell'universalità come universalizzazione delle esistenze individuali è stato Kant, sempre presente nei passaggi decisivi della teoresi piovaniana (dalle Linee di una filosofia del diritto ai Principi di una filosofia della morale, dal saggio su Perfezione e finitudine del 1977 al libro postumo del 1981 su Oggettivazione etica e assenzialismo). Con il filosofo di Königsberg e il suo criticismo è definitivo il commiato dall'ontologia, nel pensiero e nell'azione, nella gnoseologia e nell'etica. L'universalizzazione diventa legge del soggetto che si oggettiva individualizzandosi ${ }^{40}$. Sganciato dal noumenico, il fenomeno si giustifica nell'azione e con ciò il soggetto trova le origini del proprio conoscere. Il ponte verso l'universale è l'individuo stesso che nell'individuarsi nella dimensione storica, deve superare il particolarismo della soggettività irrelata. Non nell'universale astrazione, ma nel singolo che si universalizza, perseguendo i suoi ideali con tensionalità indefinita, va individuato il soggetto responsabile del nuovo

\footnotetext{
38 «L'umanesimo rifiuta di essere assoluto singolarismo, pura celebrazione del singolare come singolare, perché reca nel suo istinto ricordo di un'esperienza da cui pè germinato, l'esperienza della formazione concettuale della persona» (LFD, p. 496).

${ }^{39}$ CSCM, p. 170, 221, 66. Da questo punto di vista ha notato efficacemente V. Verra che «la ricerca di Piovani appare estremamente suggestiva non solo in campo filosofico in senso stretto, ma anche per una più ampia funzione liberatrice nel campo della cultura e della scuola» (recensione a CSCM in «Rassegna di cultura e vita scolastica», XXI, 31 marzo 1967, n. 3, p. 6). Sulla convergenza delle analisi di Leibniz e Vico si veda il commento acuto di A. Negri, Recensione a CSCM, in «Giornale critico della filosofia italiana», s. III, XXI (1967) II, p. 300.

${ }^{40}$ Cfr. G. Lissa, Anti-ontologismo e fondazione etica in Pietro Piovani, cit., p. 55 e sgg. F. Tessitore, L'«itinerario» di Pietro Piovani, cit., p. 423-424.
} 
dovere, secondo una lezione nella quale accanto al formalismo etico di Kant si colloca la filosofia del soggetto di Kierkegaard, entrambi chiamati a collaborare per la convinzione di dover rendere omaggio a «qualunque valore attesti una capacità di tensione che, oltrepassando l'immediatezza della singolarità empirica, sappia mediarla in attitudine idealizzante che sia realizzazione di un'individualità ${ }^{4}$. Si tratta di riconoscere la dinamica morale nel formalismo etico che, "mettendo in azione" la norma (kantiana), diventa «formazionismo». Il che consente di richiamare la centralità di un «sistema di forme» relativo alla loro costituzione intenzionale. In Piovani, lettore di Simmel, matura la rinuncia - perché il formalismo rimanga fedele a se stesso - alla pretesa di conoscere precettivamente i molti modi dell'ubbidire reale all'imperativo categorico, «lasciando alla storia la conoscenza possibile degli effettivi modi in cui le intenzioni morali agiscono, rettamente realizzandosi nel compimento del dovere, nell'impulso di uno sforzo tanto esemplare da poter essere assunto come modello etico universale». La «sincerità dell'intenzione», introdotta tra antico e moderno da Agostino a Pascal, resta il motivo fondante l' «individualità segreta» di ogni contenuto, nella quale può penetrare la conoscenza ricostruttrice della storia con partecipazione tanto aderente da esser in grado di conoscere «i contenuti individuali dell'infinita forma dell'universalità etica $\rangle^{42}$. Occorre, allora, individuare le fonti del dovere non in una razionalità data ma nei processi di razionalizzazione propri della logica dell'esistenza, del da farsi nelle diverse situazioni ${ }^{43}$. L'origine esistenziale dell'umanità del soggetto costituisce l'unica via d'uscita dalla passiva sottomissione a un dovere impersonale. Puntare sull'esistenza dell'individuo significa accogliere l'invito di Kierkegaard a «diventare se stessi» e aspirare all'universale che si scopre in sé. Il che consente all'analisi filosofica di trascorrere sul piano dell'esperienza morale grazie al superamento del coscienzialismo immediatistico e intuizionistico di tutta una tradizione di pensiero che, passando per Maine de Biran, giunge fino a Bergson.

${ }_{41}$ CSCM, p. 207. Sul formalismo etico nella monografia del $1961 \mathrm{cfr}$. di F. Tessitore, Tra esistenzialismo e storicismo: la filosofia morale di Pietro Piovani (1976), cit., p. 468 e gg. ${ }^{42}$ CSCM, p. 209.

${ }^{43}$ A. M. Nieddu, Normatività Soggettività Storicità. Saggio sulla filosofia della morale di Pietro Piovani, cit., p 45, sgg. e. 150 sgg. 
Se Kant ha saputo rendere autonome le coscienze in senso non coscienzialistico, negando ogni predeterminazione ai contenuti dell' azione, egli, tuttavia, può indurre a pretendere che a pensare non sia l'uomo ma l'Umanità, «una unitaria Umanità sovrastante, astratta collettività pericolosamente unificante le individualità plurime». Per tale ambiguità il formalismo può dar lievito a un rinnovato totalismo, fonte dello storicismo idealistico che assolutizza il soggetto conoscente nel Noi della ragion pratica $^{44}$. La messa in crisi dell'idea moderna di soggettività comporta anche la sottolineata insufficienza del fondamento autonomistico dell'etica kantiana e la ricerca di nuove certezze alla drammatica realtà lacerata e atomizzata dell'uomo contemporaneo. La ricerca dell'universalità nell'individuale verrà abbandonata quando sarà introdotta da Fichte la noumenizzazione del fenomeno e da Hegel l'io assolutizzato del «sapere assoluto» della filosofia dello spirito: «Piuttosto che riconoscere, con più consapevole approfondimento, che la coscienza individuale può aspirare alla verità ma non contenerla, non dominarla, la filosofia, preferisce annegare la finitudine del soggetto nella totalità onnisciente di un falso Infinito» ${ }^{45}$. La proposta si fonda sul presupposto di una natura 'sostanziale' dell'uomo e non tiene conto della dinamicità inerente al costituirsi della personalità $\mathrm{o}$, in altre parole, della storicità. Separato dalla cosa in sé, il soggetto del criticismo, mentre riconosce la capacità creativa dell'intelletto, non totalizza la soggettività nella razionalità. L'idealismo, invece, è questa totalizzazione. Lo spirito assoluto è il grande $I o$ che accoglie tutto, non può attenersi al fenomeno come fenomeno: è una filosofia dello Spirito assoluto, proprio perché ingloba il noumeno, conosce la cosa in sé e attraverso la sua mediazione, il tutto. Il fenomenico, il determinato, l'esistenziale esistono solo se sacrificati al trionfo finale del concetto previsto dal panlogismo hegeliano. Ingigantito fino al tutto, l'io non ha più bisogno di spiegarsi, chiarirsi, conoscersi come io né di stabilire $\mathrm{i}$ modi del rapporto condizionante col noumeno. Comprende in sé anche il noumeno e si ingrandisce proprio per non diversificarsene. Con la totalizzazione idealistica è frustrato ogni sforzo di desostanzializzazione

$\overline{44}$ CSCM, p. 222. Il tema è centrale nella magistrale ricostruzione di F. Tessitore, $L$ '«itinerario di Pietro Piovani, cit., p. 423 e sgg.

45 CSCM, p. 85. 
dell'io. L'idealismo eleva lo stesso io a soggetto assoluto e a sostanza, dando luogo a una vera e propria mistificazione dell'universalizzazione. La «dottrina della scienza» è una conoscenza di assoluti che, come attesta l'idealismo kantfichtiano, vuole proiettare la volontà individuale al di là di se medesima con atti di creazione che determinano la soggettività dell' Iità o dello Stato. La sua forza per valere ha bisogno di ingrandirsi: questo ingrandimento è appunto, l'entificazione della volontà nel Soggetto assoluto e nello Stato, cioè nelle due entità in cui si conclude lo sforzo di fondare tutto sulla volontà sopraindividuale e non porre l'Io individuale quale regola universale. L'esperienza del soggetto conoscente ed agente vale contro ogni totalismo che tenti di riproporre l'oggettiva totalità, contro la totalizzazione e l'universalismo che miri a restaurare l'oggettivo universale $^{46}$. Lontano dagli esiti della filosofia della storia di Hegel e da ogni restaurato storicismo assoluto è l'elogio piovaniano della filosofia moderna in quanto «negazione della filosofia del pensiero, (...) rifiuto consapevole di qualunque "filosofia dello spirito", (...) abbandono risoluto dell'orizzonte di un universo concettualistico, (...) [delle] varie forme di "storicismo assoluto", ambiziose di ricreare il totalismo in una nuova assolutezza, in cui la storia sia ricondotta al pensiero che la pensa, dunque logicizzata, nuovamente "ridotta" a filosofia, in un conclusivo monismo negatore delle libere individualità plurime ${ }^{4}{ }^{7}$.

Fedele alla lezione dello Historismus moderno e contemporaneo, Piovani assicura una prospettiva filosofica allo storicismo in senso criticoproblematico, il solo effettivo tentativo di indagare e capire per quali vie e con quali ragioni l'universale riesce a manifestarsi nella storicità

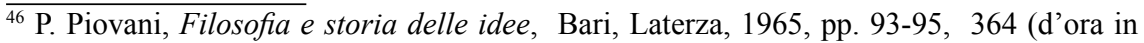
poi si cita con la sigla FSI; edizione anastatica, presentazione di F. Tessitore, introduzione di F. Lomonaco e con un saggio di G. Giannini, Roma, Edizioni di Storia e Letteratura, 2010; trad. tedesca, hrsg. von M. W. Hebeisen mit einer Einführung von F. Lomonaco, Schweizerischer Wissenshafts-und Universitätsverlag SWUV, 2010). Su quest'opera e, più in generale, sul Piovani teorico e storico della storia della filosofia segnalo i noti contributi di Cacciatore, Malusa e Marcialis, di Nuzzo e Tessitore, rinviando alle aggiornate indicazioni offerte in Bibliografia degli scritti su Pietro Piovani (1948-2000), a cura di P. Amodio, cit.; Bibliografia degli scritti di storia della filosofia di Pietro Piovani, a cura di G. Giannini, in P. Piovani, Indagini di storia della filosofia. Incontri e confronti, cit., pp. XLIX-LXI e in Bibliografia degli scritti su Pietro Piovani (2000-2007), cit.

${ }^{47}$ Così nella Nota di autopresentazione di CSCM, cit., p. 549-550.
} 
individualizzata. Esso in opposizione a ogni universalizzazione della storia si propone quale filosofia del concreto in situazione e filosofia storica dell'individuale, strettamente collegate alla curvatura teorica delle tesi di Humboldt e Dilthey, di Droysen, Troeltsch e Meinecke, quest'ultimo impegnato a cogliere quel "capire rivivendo" proprio della storicità moderna, imperniata sulle prerogative della soggettività individuale. L'indagine storica può ricostruire un'esperienza solo rivivendola e ricostruendola con chi l'ha esperita. Il conoscere storico comprende l'altro solo se sa vivere con l'altro e nell'altro. Questo vuol dire che il compito dello storico è conoscere gli altri, attraverso la comprensione e l'introspezione, quel trasferimento dell'io nel tu, teorizzato da Dilthey e riferito al compito dello storico da Humboldt e Droysen. Il vero storico prova una soddisfazione immensa nel vivere simpateticamente con gli altri uomini. Perciò non diffida dell'introspezione ma la coltiva, come insegna Renan che ha sottolineato il criterio storiografico del rispetto di ogni «âme individuelle» ${ }^{48}$, da analizzare con indagine attentamente minuziosa, espressione di una mentalità nuova che vuole sottrarsi all'uniformità e comprendere ogni forma nella mutevolezza dei modi, nella mobilità inesauribile delle esistenze. Da questo punto di vista è messa in rilievo la ricerca dei fatti della storia e delle loro ragioni, espressioni di una coscienza individuale considerata dallo storico nella sua alterità come quell'altro da sé che deve essere compreso nella sua specificità, in un movimento di idee e di fatti da non ricomporre in una sintesi assoluta e totalizzante ma da accogliere in un'esperienza autentica di vita che è autentica inquietudine.

Piovani sente molto tali esigenze teoriche con riferimento al problema della storia della filosofia. Ritornando su temi già svolti in Filosofia e storia delle idee (1965), si mostra particolarmente ostile verso quelle tipologie e generalizzazioni storiografiche che hanno espunto ogni riferimento all'individualità storicamente determinata del fatto-pensiero $\mathrm{e}$ da quella connessione di verum et factum introdotta da Vico ed enunciata come programma della nuova filosofia storica di Dilthey per ribadire il carattere individuale e storico della cultura europea, distinguendo, anche

${ }^{48}$ CSCM, p. 230, 198, 199.

49 Ivi, p. 174, 176, 54; ma sulla storiografia neoidealistica di Croce e Gentile che denuncia 
rispetto alla intransigente reazione antidealistica, posizioni come quelle di Croce e, in particolare, di Giovanni Gentile, il filosofo in quanto storico ed erudito, attento alla ricerca e allo studio dell'individuale fino al punto da equilibrare indirettamente, involontariamente la filologia del neospaventismo con il positivismo di scuola fiorentina ${ }^{49}$.

Un «esame di coscienza storiografica» è quello condotto, discutendo sulle Osservazioni preliminari a una storia della filosofia (1959) di Eugenio Garin, condivise quando la storia è prospettata come conoscenza minuziosa dell'individualeche si fa scienza con l'uso del metodo filologico, garanzia del rispetto conoscitivo «dell'alterità, che è oggettivata, così, nella particolare obiettività dello storico» ${ }^{50}$. La rivendicazione della filologia affonda le radici in un terreno filosofico, ripensando al rapporto soggetto-oggetto. La funzione della filologia, abilitata a tentare di fondare la scienza nuova della storia, si manifesta quando è impiegata originalmente dal filosofo-storico geniale a collaudare le sue intuizioni con la più completa documentazione possibile. Il dovere di conoscere con la massima esattezza possibile si fonda sul rispetto pieno dell'altro del quale si possono discernere i tratti maggiori e minori solo se tutti sono stati accertati e analizzati. Premessa e sostegno del sapere storico, la filologia è la «scienza della critica», il «metodo più atto a garantire il rigore metodico della ricerca storica $\rangle^{51}$. L'attenzione al documento filologicamente accertato non è ingenua fiducia nella lettera delle parole consegnate dalla tradizione, ma volontà di conoscere quanto più liberamente possibile quelle parole, affinché con libertà possano risuonare in chi le ascolta ricostruendole e ritrovandole solo se le abbia intraviste dentro di sé con un lavoro che è già storico, perché induzione fondata sulla conoscenza di tutta una serie di pensieri pensati e segni di

e insieme giustifica gli interessi storicistici cfr. ivi, p. 101.

50 Ivi, p. 49. «La filologia diventa così catarsi della intuizione primitiva al servizio della comprensione dell'altro, che è rispetto. La comprensione dell'altro è, nei limiti dell'umano possibile, perfetta solo se la conoscenza sintetica sia sostenuta da un'analisi che non trascuri e non disprezzi le minuzie, ma sappia che la realtà di una figura può vedersi nel suo corposo insieme solo quando tutte le sfumature siano state attentamente osservate e l'occhio non si sia soffermato solamente sulle linee più visibili» (ivi, p. 50-51). Cfr. F. Tessitore, Lo storico della filosofia (1991), poi in Id., Contributi alla storia e alla teoria dello storicismo, vol. V, cit., p. 526 e sgg. Il saggio di Garin è stato ripubblicato in E. Garin, La filosofia come sapere storico, Bari, Laterza, 1959 (n. ed., ivi, 1990), p. 55-149. 
una vita che solo uno storico riesce a ricostruire, rivivendola liberamente. Incarnando un nuovo modo di avvicinamento alle dimensioni della realtà, nelle misurazioni come nelle intuizioni, la filologia è chiamata a «indurre a un nuovo corso la storiografia filosofica» come insegnano le pagine di Spitzer di Critica stilistica e del Wilamowitz del Pasquali ${ }^{52}$. Stanno qui le ragioni che fanno privilegiare una storia storica della filosofia, intesa quale storia delle filosofie, ma senza ricondurre le plurime filosofie esistite ed esistenti al ruolo di precedenti della filosofia professata, con ciò ricadendo in un monismo metafisico di antica matrice (aristotelica), in un monistico pensiero realizzante in sé un concettuale Tutto. Di qui il rifiuto di una storia della filosofia che non pretenda di sapere alla perfezione quale sia il vero sistema della storia ma si accontenti di comprendere il significato delle presenze nella storia quale storia delle idee intrecciate al significato delle azioni umane realizzate e non strutturate idealisticamente, trascendenti l'individuale. Filosofia e storiografia, filosofo e storico della filosofia non devono mai lasciarsi sfuggire l'individualità, preferendole le generalizzazioni, comprese quelle allettanti e più insidiose interne alle metodologie della sperimentazione scientifica. La scelta individualizzante dello storico e quella generalizzante-universalizzante del filosofo debbono unirsi nello sforzo di fondazione filosofica delle scienze dell'uomo e della storia. Se la storia della filosofia non vuole correre il rischio di perdere l'individuo e l'individuale, deve rimanere fedele alle sue prerogative di storia, alla conoscenza dell'altro nella sua individualità profonda, perché ne va della sua stessa esistenza nel mondo. La storia della filosofia è una parte della cultura storica, una storia filologicamente rispettosa dell'altro e preoccupata di non sovrapporre le proprie idee a quelle degli altri. Eppure non vieta la personalità dei giudizi né impedisce il contatto tra soggetti e, quindi, ogni reale storia, distante sia dalle deformazioni soggettivistiche che dalle ingenuità oggettivistiche ${ }^{53}$.

Nella reazione all'idealistica e, per meglio dire, alla neoidealistica unicità-unità della filosofia questa proposta è davvero degna di nota per le innovative potenzialità teoriche e culturali che contiene, quando formula

51 FSI, p. 65.

52 Ivi, p. 211

53 Ivi, p. 239. Sul tema è efficace la recensione di P. Beraldi, in «Filosofia», XXII (1971) 
l'esigenza di un punto d'incontro tra il metodo scientifico della storia (che è il metodo filologico) e la metodologia delle varie scienze della natura. Tale precisazione è utile a segnalare come la storiografia filosofica piovaniana si sia orientata verso la concretezza del fare storia in cui è rilevante la funzione delle idee per legare indissolubilmente il fare storia al fare filosofia: «Titolari di quel valore universale che è la loro stessa personalità, gli uomini si estrinsecano, si realizzano in forme storiche tanto varie da essere irriducibili a un modulo concettuale unico (...). La storia dell'uomo, riportata ai suoi valori umani individuali, liberata dal megalismo onniabbracciante della segreta provvidenzialità disvelata, ammonisce che nessuno può sapere quanto valga un uomo (...) fino a che le sue azioni non siano tutte spiegate e la sua esistenza non si sia esplicata nel suo complessivo senso, non si sia realizzata entro forme istituite, in un raggio di rapporti intersoggettivi intersecantisi $>{ }^{54}$.

Ma il punto d'arrivo di queste tesi non è solo la conferma della storia storica della filosofia quale panorama di contaminazioni e di scontri, di reciproci condizionamenti tra un filosofo e il suo ambiente. Piovani non rinuncia alla specificità del taglio filosofico; può condividere la polemica antihegeliana circa la presunta subordinazione della storia alla logica, ma non la riduzione della filosofia a storia. Egli accerta, invece, la possibilità teorica di una filosofia storica fatta di idee umane alla maniera di Vico e in sintonia con la svolta anti-ontologica del pensiero moderno e con la dissoluzione della metafisica come scienza di cause prime. È il trascorrere di momenti interpretativi diversi che induce a cogliere nella storia una "nuova logica" della concretezza e a individuare l'autentica natura umana nelle potenzialità ideative del pensiero: «La storia, esortando alla comprensione di tutte le forme in cui l'uomo tende a realizzarsi, non postula una molteplicità negatrice di un'unitaria idea dell'uomo; al contrario, avverte che questa idea non vive che nelle idee dell'uomo che si sono variamente incarnate e che possano ancora incarnarsi con lo sforzo del loro vivere $(. .$.$) , in feconde lotte contro proposte diverse o antitetiche { }^{55}$.

IV, p. 502-503.

${ }^{54}$ CSCM, p. 235-236. Su questo tema cfr. A. Perrucci L'etica delle responsabilità. Saggio su Pietro Piovani, cit., p. 47 (ma cfr. anche le, p 9, 23-24, 32-33, 70-77).

55 CSCM, p. 150-151. Cfr. FSI, p. 179-180: «Nella storia l'idea è vista quale attitudine 
Nell'aggiornato esercizio storiografico la storia della filosofia rimane se stessa, se vede effettivamente le idee saldate alle cose e non presunte ideali realtà esterne in nome di un conoscere che rinunci al suo compito. Le idee sono in rebus humanis solo se si ammette la loro capacità di capire le cose, trasformandole con lo stesso capirle e interpretarle. Esse rappresentano lo spettro delle tensioni umane nella temporalità in cui sperimentano l'invincibile scarto tra il datum e l'idealità, tra forze plurime di esistenze storiche, ricondotte a una «fenomenologia della genesi», perché nel pensiero moderno sempre più profondo è il convincimento che «la natura delle cose non possa comprendersi meglio che nel loro nascimento» ${ }^{56}$.

Per Piovani non è possibile rendersi conto dello storicizzarsi delle idee, se non si prende atto che ogni realizzazione dell'individualità implica un'idealizzazione. Questa impostazione del binomio idearealtà rappresenta la fine di ogni cosalizzazione o localizzazione delle idee. «L'idea non si identifica con il fine di una direzione perché in una simile direzione sarebbe raggiungibile, mentre l'energia da cui ricava tutta la sua forza motrice risiede nella sua inattingibilità» ${ }^{57}$. Proprio in tale polemica acquista significato la funzione della filosofia come unificazione delle esperienze anche irrazionali radicate nell'esistenza dei pensatori; una filosofia che è pensiero vissuto vivente, razionalità ricca di capacità intuizionali (Bergson) e priva delle astrattezze del razionalismo puramente intellettuale. Tale diagnosi è coerente con le ragioni della distanza del Piovani dallo storicismo (integrale) gariniano e dai riferimenti alla concezione marxistica della storia, dalle concessioni ai «teorici del

ideativa dell'uomo. Scompare ogni datità più o meno innatistica: l'idea altro non è che l'ideazione, storicamente spiabile nei suoi palesi eppure incerti effetti aperti alla molteplicità delle conseguenze imprevedibili e delle interpretazioni possibili. Se il pensiero si convince ormai che la vera natura dell'uomo è la storia dell'uomo, la vita si presenta come una permanente creazione di forme ideali in perenne movimento. (...) Una logica siffatta può accettare di essere tanto inoggettiva (per dir così) da comprendere come si possa sostenere che gli uomini più che avere delle idee sono delle idee, giacché essi non hanno che le idee che vivono: la presenza di idee nella storia è conoscibile solo se esse siano coerentemente, interamente vissute».

56 FSI, p. 279-280. «Le idee sono (...) formazioni concettuali umane, nate nell'opera dell' uomo creatore delle sue varie civiltà, progredienti e insoddisfatte: sono forme dell'umanità in fieri, la quale non è un dato da descrivere, ma un progresso da seguire nella mobilità delle sue varie individuazioni» (CSCM, p. 145). 
pensiero come pensato in società, quasi come pensato sociale» con un condizionamento deterministico della società sulla filosofia e un coerente «conformismo della ideologia» dello spirito del tempo, sintonizzato sulle seducenti ma inaccettabili tesi del Mannheim, fautore di una «nuova teologizzazione della Storia in un Collettivo» in cui l'individuo fagocita a vantaggio della «determinante Società $»^{58}$.

I rilievi critici sui contrassegni positivistici del marxismo di Garin e sulla conseguente separazione del fatto dalla teoria e dal valore motivano la scelta piovaniana di abbandonare le premesse e gli esiti della meditazione sul diritto, interpretato sulle orme del Capograssi come «azione dell'azione», «salvezza dell'azione». La crisi della normatività è ciò che la ricerca filosofica sul diritto ha vissuto e che le pagine piovaniane dalla fine degli anni Cinquanta riflettono. La filosofia del diritto è quasi ripudiata per un bisogno di fedeltà alla concreta comprensione storica con lo scopo di teorizzare un più adeguato impegno etico dopo gli esercizi teoretici dedicati ai temi del giusnaturalismo.

Non a caso la fondamentale opera del 1961 su Giusnaturalismo ed etica moderna si chiude con alcune pagine di Kierkegaard tratte da Aut-Aut e assunte a modello esemplare della moderna coscienza individuale ${ }^{59}$. Se l'etica moderna è rispettosa delle vocazioni individuali, il giusnaturalismo è, al contrario, una teoria legalistica che, includendo l'individuo nell'ordine cosmico, lo costringe a legittimare il suo agire in termini di adequatio a una natura universale, di obbedienza a un disegno finalistico dell'intero universo. Il compito di una reazione antigiusnaturalistica è quello di emancipare la filosofia da tutte le nostalgie e venature (gius) naturalistiche presenti nella modernità, quasi timorosa, a volte, di abbandonare gli ultimi residui dell'ordine cosmico-naturale, infranto al sorgere dell'età moderna.

\footnotetext{
${ }^{57}$ FSI, p. 188.

58 CSCM, p. 46, 42, 43. Cfr. M. T. Marcialis, Piovani storico della filosofia, in «Archivio di storia della cultura», XIV (2001), p. 217 e sgg. (anche in "Giornale critico della filosofia italiana», LXXX, 2001, I, p. III, p. 33-35).

59 P. Piovani, Giusnaturalismo ed etica moderna, Laterza, Bari, 1961, nuova edizione a cura di F. Tessitore, con due note di N. Bobbio e G. Calogero, Liguori, Napoli, 2000, p. 119-120 (d'ora in poi si cita con la sigla GEM; trad. tedesca, hrsg. von M. W. Hebeisen mit einer Einführung von G. Cacciatore und von F. Tessitore, Schweizerischer Wissenshafts-
} 
Il rifiuto del giusnaturalismo spiega le ragioni della filosofia morale di Piovani che non poteva non approdare a un'intrinseca morale storicistica. Infatti il riconoscimento della conoscenza storica come conoscenza delle individualità in tensione doveva necessariamente includere la moralità di questa natura storica dell'uomo in coerenza con l'antintellettualismo e l'antilogicismo, l'anticosmologismo e l'antiuniversalismo consolidati. L'essenza di ogni realtà fenomenica è di non riuscire mai ad esistere integralmente; non si realizza mai compiutamente, perché è solo in quanto tende ad essere quale dovrebbe essere, secondo quella tensione etica, la cui perdita può comportare la ricaduta dell'inumanità, giacché «la civiltà non è datoacquisito definitivamente, madelicatissimocomplessodicondizioni, che sta alla fatica costante e rinnovantesi degli uomini conservare e migliorare ${ }^{60}$.

Nella critica storicistica alla razionalità metafisica occidentale e nella presa d'atto della definitiva dissoluzione di ogni assolutismo tanto naturalistico quanto idealistico stanno i presupposti della fondazione di uno storicismo etico. Con ciò si compie il discorso contenuto nell'ultimo saggio che dà il titolo al libro e che delinea i contorni di uno storicismo nuovo ed originale a partire dal rapporto etica-storia con al centro la moderna filosofia dell'individualità; una filosofia che è sforzo continuo di ritrovare le forme storiche dell'espansione e della realizzazione della personalità individuale. Quando quest'ultima si scopre universale e sa di essere insieme individuale ed universale, l'etica si traduce in realtà: «Appunto la dimensione della storicità attesta che l'individuo o si personalizza, liberandosi dall'accidentalità irrelata, o non è. In questo senso, la storia è il caratteristico banco di prova della intrinseca moralità di questo valore». In una certa situazione storica l'universale è tensione valorativa, quindi attività formativa della personalità in continua espansione e perfezionamento, implicando un'effettiva dialetticità, concretatasi nella trasformazione delle forme storiche, nella precisazione che la «storicizzazione dell'individuale è chiarimento della verità secondo cui non c'è individualità espansa e attiva se non c'è sviluppo di personalità, correlazione, incontro inter-personale, effettiva de-singolarizzazione» ${ }^{61}$.

und Universitätsverlag SWUV, 2010 II. Abteilung, Band 6).

${ }^{60}$ CSCM, p. 175. 
Approntati gli strumenti logici e metodologici che consentono di penetrare l'intimità della coscienza grazie alla loro coerenza con la vita del soggetto - che è vita storica contestualizzata e individualizzata in una specifica esistenza - diventa possibile pensare alla costituzione di un'etica dell'individualità. Così lo storicismo critico contribuisce a incrementare la sola forma di civiltà in cui l'uomo lascia «il segno della propria volontà d'infinitarsi» ${ }^{62}$. La possibilità di una «morale storicistica come fenomenologia degli ideali dell'uomo», argomentata nell'ultimo capitolo dell'opera, si afferma quando cade ogni residuale estraneità tra conoscenza storica e coscienza morale, quando dopo Kant si è scoperto che l'universale non è estraneo alle concretizzazioni storiche della vita umana. Lo storicismo nella versione critica non merita «l'accusa di sostanziale immoralismo», perché la rifondazione dell'individualità passa dal rifiuto della chiusura singolaristica alla fondazione della moderna storicità: "Tanto le ragioni della conoscenza storica non contraddicono le ragioni della coscienza morale che addirittura lo slancio conoscitivo della storia può essere simile a quello della carità, per natura sua pronta a comprendere qualunque azione ${ }^{63}$. Nell'universale che sa di esistere nell'individualità è possibile riscattare 1 'originaria difettività esistenziale e, insieme, raggiungere la legittimità etica e filosofica della permanente tendenza dell'uomo a esprimere forza creatrice e rinnovatrice delle idee nella storia: «L'universale vive nell'individuale che ne garantisce l'infinità, perché la logica dell'individualità richiede che l'esistenza individuale non possa cercare di realizzare se stessa senza ubbidire al bisogno d'infinito che reca in sé e di cui fa esemplare attestazione» ${ }^{64}$. Al fondo è la ridefinizione dell'etica come scienza della storia che collauda le libere volontà indidivuali. La costatazione della libertà esalta l'individualità fondandone la responsabilità e, grazie alla approfondita teoresi, induce Piovani a interrogare la "natura imperfetta" dell'uomo. Questi è tanto più angosciato per tale imperfezione quanto più comprende che il suo destino sta nel coraggio di tale ammissione, in grado, paradossalmente, di contribuire a contrastare la lesione. Il male consiste proprio nel mettere in discussione l'accettazione vitale, nell'ostacolare l'autentica esperienza

${ }^{61}$ Ivi, p. 170, 181.

${ }^{62}$ Ivi, p. 210.

${ }^{63}$ Ivi, p. 161, 157, 224. 
di vita di tutte le individualità nella storia. Il vero collaudo della storicità prima di far avvertire la libertà dell'uomo nasce dall'avvertire la presenza della realtà del male cui Piovani riconduce la tentazione singolaristica. Questa conclusione pone alla filosofia morale rilevanti e delicatissimi problemi teoretici e storici quali quelli del progresso e del perfettismo che introducono alla gran questione della teodicea, argomentata e sviluppata a partire dalla declinazione "sociale" del tema in Rosmini ${ }^{65}$.

A legittimare la dimensione etica dello storicismo piovaniano è la teorizzata connessione tra la concezione critica della storicità e il problema dei valori. Con Troeltsch si comprende il relativo dei valori in rapporto ad un assoluto contenuto in essi, giacché «la relatività dei valori ha senso se in questo relativo viva qualcosa di assoluto (...); l'assoluto della visione storicistica è l'individuale nella sua segreta originarietà, nella sua potenza di sviluppo imprevedibile ${ }^{66}$. L'attenzione al relativo nello Historismus è espressione di uno storicismo che rompe con l'universalismo tradizionale e un ordine erede di quello teologico. All'individualità «è relativo il relativismo della storia», perché essa vuole essere coerente con il significato di una storicità non più totalità contenente un contenuto o valore da dedurre, non più logica dialettica ma effettiva che «non è della storia, ma nella storia, giacché si concentra nella dinamica di trasformazione delle forme storiche», sugli esiti non scettici ma prospettivistici del relativismo storicistico. Insieme di individualità viventi e soggetto di valori, la storia non vale mai come un tutto inglobante, ma per le energie che include per le responsabilità richieste da un progresso solo e sempre possibile della civiltà. Delle connessioni storiche si dà una ragione unitaria, vista dal Piovani non nel fatto che la storia sia storia della totalità e assegni un posto predeterminato agli individui, bensì nel senso che «la dimensione della storicità attesta che l'individuo o si personalizza, liberandosi dall'accidentalità irrelata, $\mathrm{o}$ non è̀ ${ }^{67}$.

\footnotetext{
${ }^{64}$ Ivi, p. 179.

${ }^{65}$ Mi riferisco, naturalmente, alla monografia piovaniana su La teodicea sociale di Rosmini, Padova, Cedam, 1957 (II ed., permessa di F. Tessitore con postfazione di G. Cantillo, Brescia, Morcelliana, 1997). Sul tema sono utili le pagine di F. Tessitore, Pietro Piovani e la questione della teodicea, in "Archivio di storia della cultura», XIV (2001), p. 5-26.

${ }_{66}$ CSCM, p. 162. Sul tema sono fondamentali gli studi di G. Cacciatore, L'etica dello storicismo, Lecce, Milella, 2000, cap. VI su «L'etica storicistica di Pietro Piovani», pp. 125-196; cfr. anche Id., Etica dello storicismo e filosofia pratica nel pensiero di Piovani, in «Archivio di storia della cultura», XIV (2001), p. 33-34.

${ }^{67}$ CSCM, p. 171, 207, 177, 170.
} 
Il significato del relativo è, dunque, il significato stesso della vita senza inibire l'esigenza dell'assoluto che vive e opera in noi, innalzandoci al di sopra di noi stessi. Con Laberthonnière Piovani coltiva il senso del relativo per comprendere l'individualità del passato e preparare il futuro. La cura del relativo è il rifiuto di una storia che elida l'accidentale; è la rivendicazione dell' «empirico situazionale», «piattaforma allo slancio dell'individualità». L'empirico stesso non è il singolare irrelato, il particolare chiuso all'alterità, ma la nota individuale che si sottrae ad ogni fagocitante totalità per affermare la propria tensione ad agire nello sviluppo dinamico dell'esistere ${ }^{68}$. In effetti, ben più scandaloso e pericoloso del relativismo storicistico sarebbe un «automatico immobilismo dei valori», il che finirebbe col compromettere la stessa libertà umana, senza la quale si distruggerebbe l'idea di moralità. Nella mutevolezza dei molteplici valori la sola costante necessaria all' esistenza di tale idea è l' «esigenza» stessa dei valori $^{69}$. La lezione del relativismo storicistico non è, allora, predicazione di nichilismo moralmente inconcludente; è la critica costruttiva di valori non intangibili, vissuti nella consapevolezza che permanente è l'aspirazione umana a crearli. E tutto ciò perché le individualità sono energie viventi, non determinazioni provvisorie ed evanescenti di un divino spirito del mondo. Questo è l'unico "presupposto assoluto" da riconoscere per coniugare in modo corretto etica e storia. Comprenderne il nesso fondamentale significa concepire il dover essere non nell'astrtta staticità dei princìpi formali e aprioristici, ma nell'individuazione delle forme e dei contenuti della soggettività costitutivamente storica che agisce e sceglie. Su questa base Piovani può assumere pienamente i fermenti e le inquietudini di un'etica definita forse più dalle sue incertezze che dalle sue sicurezze; può addentrarsi entro le complesse pieghe del nichilismo contemporaneo senza lo sgomento, il pathos che avvolgono buona parte del cosiddetto pensiero negativo. Rifiutato ogni sostanzialismo, criticato ogni ontologismo, i valori vengono radicalmente restituiti alla loro storicità: essi valgono nella misura in cui sono storicamente vissuti, cioè creati, formati, scelti dagli individui che

\footnotetext{
${ }^{68}$ Ivi, p. 166, 165. «L'individuo non è individuato una volta per tutte: non fa che individuarsi. La naturalità del suo essere esiste solo per essere riscattata grazie all'attività dell'individuo stesso, che non può essere un mero fatto, che non può non farsi (...): imprevedibile, l'individuo non è un prodotto, ma un crearsi: la datità del suo originario essere nel mondo non è che l'avvio a un sistema di creazioni, senza di cui l'individuo non è se medesimo»» (ivi, p. 165).

69 Ivi, p. 174, 175, 160.
} 
vivono in società. L'universalità dei valori sta nella tensione universalizzante dell'individualità nell'azione etica, esistenziale e interpersonale. Nell'importante conclusione è il riconoscimento di un solo valore immutabile, l'idea stessa del valore in quanto idea individuata, identificata nell'uomo stesso che dà valore al valore in quanto soggetto e fonte della sua storia, unico universale concreto, capacità immanente e costante di trascendimento sia pure sempre in fieri. Insomma l'individualità alla fine non può essere presa quale puro essere individuale dell'uomo, implicando la sua struttura distinzioni (tra animalità e spiritualità, singolarità e universalità); e sono distinzioni qualificanti la fisionomia dell'individuale che, per perfezionarsi, deve emanciparsi dalla «naturale animalità della storia», a conferma che etica e storia non si escludono secondo la diagnosi della diltheyana «analisi della coscienza morale» ${ }^{70}$. E, tuttavia, resta problematica la relazione tra l'individuale che è «un assoluto mai assolutizzabile» e il valore che è valorazione sempre ulteriore. Il discorso può essere trasferito sul piano gnoseologico a proposito della verità, definita «aspirazione alla verità» ${ }^{71}$, irraggiungibile nella sua interezza. Se «la verità non è le verità $\rangle^{72} \mathrm{e}$ il verum è sempre un oltre, non si può sostenere che ogni concezione sia la verità, la cui scoperta è sempre solo parziale, identificabile nella forma attingibile dall'umano che è quella della verità individuale e storica. Pertanto, anche quando la ricca e originale ricognizione storiografica piovaniana si sofferma sulle pagine di Bultmann non fa concessioni a un antico o rinnovato provvidenzialismo. In coerenza con la teorizzata connessione tra conoscenza storica e coscienza morale, il filosofo e storico delle idee interpreta la relazione tra storia ed escatologia

\footnotetext{
${ }^{70}$ Ivi, p. 170, 161.

${ }^{71}$ Ivi, p. 152, 161.

72 In una lettera del 27 gennaio 1967 a Claudio Vasale, acuto studioso di Capograssi, Piovani osserva che «la verità non è le verità: le verità come tensioni la testimoniano, esigenzialmente, nella storia, ma come tensioni proprio perché non sono la verità che è sempre oltre, e perciò non nega il mistero. Ma anche di fronte a ciò è necessaria una personale decisione, sulla quale io ho preso posizione con nettezza a p. (per me importante) 195» (lettera in copia, ora in Archivio della Fondazione "Pietro Piovani per gli Studi Vichiani", c. 1). Ringrazio il mio Maestro, Fulvio Tessitore, Presidente della suddetta Fondazione, per avermi autorizzato alla consultazione di questa documentazione inedita (e dell'altra qui di seguito utilizzata), concedendomi, altresì, l'onore di avviare i lavori preparatori alla raccolta e sistemazione del carteggio piovaniano. Cfr., da ultimo, La Biblioteca della Fondazione Piovani. La Collectio Viciana, a cura di P. Annunziata, intr. di F. Lomonaco, presentazione di F. Tessitore, Napoli, Liguori, 2005, in particolare parte V «Carte e Documenti di Pietro Piovani», p. 137-153.
} 
nel senso che essa possa risultare salvifica per le azioni individuali che hanno incontrato nella storia il divino, a sua volta umanizzatosi nella temporalità del verbum incarnato, lontano da ogni cosmologia naturale e/o sovrannaturale: «Il Progresso non ha più bisogno di imitare, o di parodiare, la Provvidenza, perché è la storia stessa che, individualizzata nell'esistenza responsabile di ognuno, si presenta come capace di farsi escatologia, per la salvezza dell'individuo che incontri nella storia il Dio che, scandalosamente, ha incontrato la storia, in un incontro che non è un mero accidente disordinante il classico processo naturale e sovrannaturale del cosmo (...): incontrato nella storia il Cristo, deve l'individuo decidere di misurarsi con lui, scegliendolo o rifiutandolo» ${ }^{73}$. Per riscattare il fatto dell'esistenza, la datità stessa dell'individuo, l'istanza "fondativa" si sposta dalle iniziali posizioni di Normatività e società alle aggiornate analisi sull'individuale che si rivela universale nel proprio mondo storico. In esso quell'istanza, trasformata in una moderna esigenza di fondazione dell' azione individuale, trasferisce la questione ontologica dalla norma alla storia $^{74}$. Ma lo fa assumendo in senso critico l'impostazione tradizionale, contaminata dalla lezione di Pascal e dalla "fondazione di una metafisica dei costumi" di memoria kantiana, misurata dalla responsabile scelta individuale nella sua tensione universalizzante.

Data de registro:28/10/2012

Data de aceite:28/01/2013

73 CSCM, p. 184, 213, nota, 195-196. Pertanto non mi sembra convincente la critica del Negri al Piovani che, a suo giudizio, «cade nell'uso della categoria del 'comprendere'» e paradossalmente giunge all'esclusione di ogni individuale per un suo presunto «atteggiamento contemplativistico di fronte all"oggetto». La teoria piovaniana delle idee che regge la conoscenza storica e la coscienza morale è proprio la messa in crisi dell'antico atteggiamento contemplativo (aristotelico), lontano, perciò, anche dal rischio paventato dall'interprete di approdare al «mare dell'oggettività» in cui può naufragare una «dialettica che ama di sviscerato amore tutto il reale» con gravi conseguenze (il conformismo) sul piano etico (A. Negri, Recensione a CSCM, cit., p. 303-304).

74 Non condivisibile mi sembra l'interpretazione del Crippa che ha visto nella dimensione etica dell'individualità «l'affermazione di una consistenza ontologica di questa (...), il parametro valutativo che orienta e condiziona l'operare in cui si attua l'individualità radicandosi ed esprimendo questa stessa e la sua tensione costitutiva al valere che è insieme consistere di quella realtà che valuta e sceglie, che stringe e raccoglie queste scelte e valutazioni, e perciò stesso, in intensità di proposizione non solo operativamente ma ontologicamente sussiste» (R. Crippa, Recensione a CSCM, cit., p. 335). 